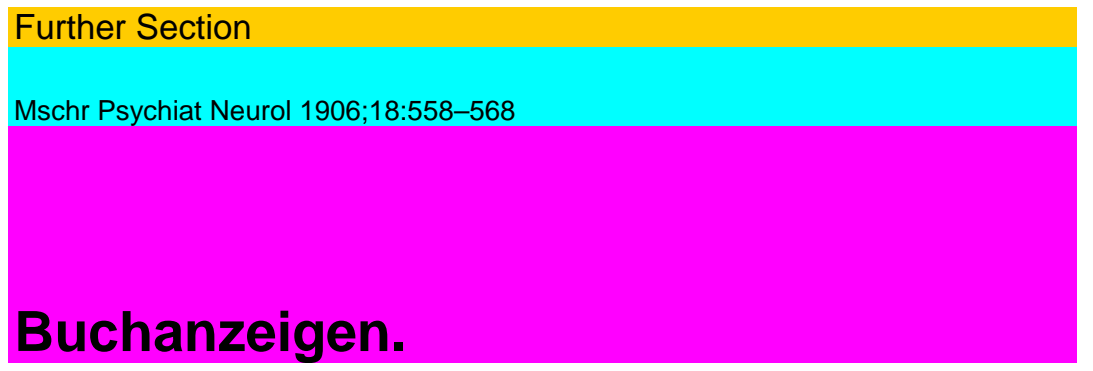

\title{
B uchanzeigen.
}

Binswanger, Otto: Die Hysterie. Wien. 1904. A. Holder.

Besprochen von Dr. Alfred Saenger, Spezialarzt für Nervenkrankheiten am Allgemeinen Krankenhause Hamburg-St. Georg.

In verhältnismässig kurzer Zeit haben wir nach den zwei grossen Monographien über die Neurasthenie und Epilepsie von dem Jenenser Neurologen und Psychiater eine Bearbeitung der Hysterie erhalten, die wagen der Gründlichkeit, des Umfanges und der Beherrschung des Stoffes geradezu mustergültig genannt werden darf.

Binswanger ist als Psychiater und Neurologe ganz besonders dazu prädestiniert, dieses so schwiSHÄ Kapitel der Neurologie und Psychiatrie erschöpfend zu bearbeiten, da er nicht nur über das grosse Material der Jenenser Irrenanstalt verfügt, sondern als gesuchter Konsulent auch eine ausserordentlich grosse Erfahrung ÜBF'der neurolosfeshen Präzis gesa(|Biii\& hat, die sich speziell auf die Patienten der höheren Stände bezieht. Letzterer Umstand ist besonders bei der in Rede stehenden Neurose von nicht zu unterschätzender Bedeutung. 
Buchanzeigen. 
Der Autor leitet sein Werk mit den Worten ein, dass die Hysterie das Schmerzenskind der Nervenpathologie sei, indem nicht nur im Laufe der vergangenen Zeit, sondern auch heute noch keine Verständigung über das Wesen der Hysterie erzielt worden sei.

Ein kurzer historischer Ueberblick von Seite $1-6$ beweist dies.

Nach Binswanger besteht der einzige bleibende Gewinn der neuesten Forschungen in der Erkenntnis, dass alle hysterischen Krankheitserscheinungen in unlösbaren Beziehungen stehen zu Störungen'der Hirnrindenfunktionen. Er hebt hervor, wie wichtig die Berücksichtigung der Individualität ist, so dass man versucht sei, zu sagen: „so viele Individualitäten, so viele Variationen der hysterischen Zustandsbilder."

Bei der sogen, hysterischen Veränderung der Grosshirnrinde stehen auf der untersten Stufe die pathologisch gesteigerten Gefühlsreaktionen, welche die ergiebigste Quelle für die Entwicklung darstellen. Sie charakterisieren sich als hysterische Affektentladungen, welche sich durch das Missverhältnis zwischen Intensität des auslösenden Reizes und der Mächtigkeit des Affektes auszeichnen

Die Ursache dieser pathologischen Affektreaktionen liegt nach Bins-wanger in einer gestörten Dynamik der kortikalen funktionellen Mechanismen, die entweder in Hyper- oder Hypo(resp. A-)funktion zutage treten.

Am sinnfälligsten werden die psychischen Vorgänge betroffen:

1. in Form von Empfindungsstörungen,

2. in Form von Bewusstseinsstörungen(Bewusstseinslücken, Dämmer-hypnoide Zustände,

Wachträume, Amnesien'),

3. in Form von Störungen der Ideenassoziation.

Als wichtigsten Punkt der Binswangerschen Auffassung der Hysterie möchte Referent hervorheben, dass es elementare hysterische Krankheitssymptome (sensible, sensorische Anästhesie, konzentrische Gesichtsfeldeinschränkung) gibt, die ganz für sich allein, gewissermassen losgelöst von jedem psychischen Elemente, bestehen können.

Er betrachtet ebenso wie Referent diese Krankheitssymptome als Kennzeichen einer unterwertigen Erregbarkeit funktionell zusammengehöriger Rindenelemente, durch welche die physio-psychologischen Bedingungen für das Zustandekommen von Bewusstseinsvorgängen, d. h. hier Empfindungen, aufgehoben sind.

Von den eben erwähnten Gesichtspunkten kann man zwei Kategorien hysterischer A nä s th e si e n unterscheiden: bei der einen besteht ein Minus kortiko-sensibler Erregbarkeit, bei der anderen eine Mehrleistung psychischer Arbeit.

Alle hysterischen Krankheitserscheinungen stimmen aber darin überein, dass jedes oymptom durch psychische Phänomene reproduziert werden kann, auch wenn bei seiner erstmaligen Entstehung ein Bewusstseinsvorgang nicht wirksam gewesen ist.

Dies ist dasjenige Krankheitsmerkmal, welches die Hysterie vor allen anderen Nervenkrankheiten auszeichnet, die gesteigerte Suggesti-bilität.

Der Verfasser führt dann noch in bemerkenswerter Weise den Nachweis, dass es unmöglich sei, alle hysterischen Krankheitsvorgänge auf eine psychologische Grundformel zurückzuführen. Er weist daher die diesbezüglichen Versuche von Möbius, P. Janet und S ollier zurück, in ganz ähnlicher Weise, wie ich es in meiner Arbeit: Hysterie und Neurasthenie bei Kindern (Verlag von S. Karger) getan habe. B. stellt sich vollständig auf den Boden, der von Ziehen in seinen physio-psychologischen Vorlesungen vertreten worden ist, dass nur dasjenige, was unserem Bewusstsein gegeben ist, als psychische Erscheinung vorhanden sei.

Die hysterische Veränderung wird von B. dahin definiert, dass die gesetzmässigen Wechselbeziehungen zwischen der psychischen und materiellen Reihe gestört sind, und zwar in doppelter Richtung; auf der einen Seite fallen für bestimmte Reihen materieller Rindenerregungen die psychischen Parallelprozesse aus oder werden nur unvollständig durch jene geweckt; auf der anderen Seite entspricht einer 
materiellen Rindenerregung ein Uebermäss psychischer Leistung, das die verschiedenartigsten Rückwirkungen auf die gesamten Innervationsvorgänge, die in der Rinde entstehen oder von ihr beherrscht werden, hervorruft.

$B$. nennt die Hysterie eine Psychoneurose $\mathrm{Z}<\mathrm{JT}$ ' ifo\% $\mathrm{i}$ ly und hebt noch hervor, wie häufig sie im Verein mit organischen Erkrankungen des Zentralnervensystems (z. B. der multiplen Sklerose) vorkommt. Praktisch am bedeutsamsten ist die Kombination mit Neurosen, spez. mit der Neurasthenie. Nach seinen Beobachtungen hat die Hälfte der Kranken die Krankheitserscheinungen der Hysterie und Neurasthenie dargeboten.

B. polemisiert hierbei gegen Möbius, der die Neurasthenie für eine von deiv Hysterie gänzlich verschiedene Krankheit erklärt, hat; B. sagt im Gegensatz zu ihm, dass viel häufiger die hysterische Veränderung auf dem Boden der neurasthenischen Dauerermüdung resp. Erschöpfung zustande kommt. Er hält auch den von der Char cot sehen Schule aufgestellten und verteidigten Lehrsatz, dass die Hysterie nur auf dem Boden der erblichen Prädisposition entstehe, für nicht zutreffend. Ebenso lehnt er die Fcresche Ansicht ab, das pathologische Prinzip der Erschöpfung auf alle Fälle von Hysterie zu übertragen.

In ähnlicher Weise tritt B. für die Kombination der Hysterie mit der Epilepsie ein, was ja von vielen Autoren noch bestritten wird.

Zum Schlüsse des Einleitungskapitels bespricht B. noch den hysterischen Anfall, dessen Entstehungsbedingungen und endlich noch die Mischformen der hystero-epileptischen Anfälle. Das Beweismaterial für seine Auffassung wird in einem späteren Kapitel gebracht.

Ein Affektshock kann bei prädisponierten, konstitutionell-hysteropatho-logischen Individuen nicht nur den ersten hysterischen Anfall, sondern auch eine Reihe hysterischer Krankheitssymptome hervorrufen, welche den Anfall für kürzere oder längere Zeit überdauern.

Mit Recht weist B. auf die traumatische Hysterie durch Blitzschlag hin, als Beweis, wie ungenügend die Definition der Hysterie als eines lediglich psychogenen Leidens ist.

Das 2. Kapitel enthält die Aetiologie, bei welcher B. die ursächlichen Momente für das Gesamtleiden von denjenigen für die einzelnen Krankheitsäusserungen auseinanderhält. Die Hauptrolle bei der Hysterie spielt die neuropathische Belastung. Dabei sind die acquirierten Keimschädigungen mit den wahren ererbten Keimänderungen hinsichtlich ihrer Wirkungsweise auf die Anlage der Frucht auf gleiche Linie zu stellen. Solche Keimesschädigungen entstehen infolge von chronischen Intoxikationen, von Infektionen, von konstitutionellen Erkrankungen und von lokalen Erkrankungen der keimbildenden Apparate.

Sehr lehrreich sind die Angaben über den Einfluss einer schweren erblichen Belastung auf die Entwicklung, die Gruppierung der Symptome und auf den Verlauf. Auf die. körperlichen Degenerationszeichen legt Verfasser geringeres Gewicht. Er ist der Ueberzeugung, dass eine scharfe Trennung der Hysterie von anderen funktionellen Nerven- und Geisteskrankheiten nur bei den Fällen mit einfacher, nicht aber bei denjenigen mit degenerativer Vererbung möglich $\ll \S$, eine Ansicht, der Referent auf Grund seiner Erfahrungen durchaus beistimmt.

Ausgezeichnet ist die Schilderung der krankhaften Züge auf nervösem, resp. geistigem Gebiet, welche unter dem Einflüsse einer schweren erblichen Belastung schon in der Kindheit oder wenigstens während der Pubertät nachzuweisen sind, wie die gesteigerte Konvulsibilität, Störungen der Gefäss-innervation (Migräne), Störungen des Schlafes, verlangsamte körperliche und geistige Entwicklung, Störungen der Empfindungen und der Gefühle. (Referent Bat in seiner Schrift über Neurasthenie und Hysterie der Kinder spezieller sich mit der Entwicklung zur Hysterie befasst, was leider B. entgangen zu sein scheint).

Sehr eingehend werden die ätiologischen Momente wie die akuten und chronischen Intoxikationen, die chronischen Infektionskrankheiten, die Stoffwechsel- und Bluterkrankungen besprochen. Alle die genannten ätiologischen Momente, zu denen noch die geistigen Überanstrengungen, erschöpfende 
Buchanzeigen. 
Einflüsse (Onanie) gehören, treten an Bedeutsamkeit weit in den Hintergrund gegenüber den schädigenden Einwirkungen der Gemütsbewegungen. Eine höchst interessante Krankengeschichte, die die Bedeutung des Affekt-shocks klar illustriert, ist auf Seite 67 mitgeteilt. An den Schluss der ätiologischen Betrachtungen stellt B. das Trauma und weist auf die Verdienste der Charcotschen Schule hin, die auf die Auffassung der Hysterie den weitgehendsten Einfluss erlangt haben; jodoch weist die Lehre nach B.s Ansicht nach verschiedenen Richtungen hin Lücken auf. So ist es nicht nötig, jedesmal eine traumatische Suggestion anzunehmen, da auch ohne jede Vermittlung eines bestimmten Vorstellungsreizes ausschliesslich durch die Affekte auf motorischem, vasomotorischem und sensiblem Gebiete die ausgedehntesten bahnenden und hemmenden Einwirkungen stattfinden können. Eine affektbetonte Empfindung hat den gleichen Wert wie die vielfach hypothetische traumatische Suggestion.

B. stimmt mit Op p en h e i m überein, dass in vielen Fällen die Molekularschädigung der zentralen Nervenelemente eine der wesentlichsten Entstehungsursachen des traumatischen Nervenleidens ist.

Der Autor fasst seine Ansicht dahin zusammen: das Trauma ist eine sehr häufige Ursache der Hysterie. Es wirkt vornehmlich auf folgende Weise Hysterie erzeugend:

a) durch die mit dem Trauma verknüpfte Affekterschütteruug und besonders bei den sog. lokalisierten Störungen durch bestimmte, durch das Trauma ausgelöste Vorstellungen (traumatische Suggestion).

b) durch die mit vielen Traumen verknüpfte Molekularerschütterung des Zentralnervensystems (Commotion), bei welcher die Molekularschädigung der Grosshirnrinde die Hauptsache ist;

c) entweder wirken beide schädigende Faktoren bei der Entstehung der traumatischen Hysterie zusammen, oder es löst der psychische Faktor für sich allein bei schon disponierten Individuen die Hysterie aus.

Die Symptomatologie wird durch sehr lesenswerte psychologische Vorbemerkungen eingeleitet. B. ist der Ueberzeugung, dass die künftige Entwicklung der Hysterieforschung eng mit psychologischen Untersuchunge verbunden bleiben muss, wenn in das Chaos der Krankheitsbedingungen und Krankheitserscheinungen Klarheit gebracht werden soll. Die gesteigerte Gemütsreizbarkoit und die krankhafte Leidenschaftlichkeit sind von altersher 'als wesentlichste Kennzeichen der Hysterie auf psychischem Gebiete betrachtet worden. Die Gefühlsreaktionen sind ein Gradmesser einerseits für das Maass und die Ausdehnung der der Hirnrinde zuüiessenden und in ihr verarbeiteten Erregungen, andererseits aber auch für den Erregbarkeitszustand der zentralen Nervensubstanz. Die Stärke der Gefühlsreaktion wird, wenn wir von individuellen Verschiedenheiten absehen, um so grösser sein, je mannigfaltiger, gehäufter und intensiver die zufliessenden Reize sind oder aber, je erregbarer die Hirnrinde ist. Als Affekt bezeichnet B. jede Gefühlsreaktion, welche einen Einfluss auf geistige und körperliche Vorgänge ausübt. Die Anomalien der Gefühlsreaktionen finden ihren klinischen Ausdruck

a) in einer krankhaften Steigerung der Gefühlsreaktion,

b) in einer Verringerung und

c) in einer pathologischen Labilität derselben

Für besonders wichtig hält Referent Binswangers Ausführungen betreffs der körperlichen Begleit- und Folgeerscheinungen der Affekte. In Anbetracht, dass das Wesen der Hysterie in letzter Linie in einer krankhaften Verschiebung der physiologischen Wechselwirkung zwischen den materiellen Hirnrindenerregungen und der psychischen Parallelreihe beruht, ist die klinische Tatsache erklärlich, dass alle psychischen Vorgänge eine abnorme Einwirkung sowohl hinsichtlich der Intensität, als auch der Ausbreitung auf die kortikalen und infrakortikalen zentralen Nervenmechanismen ausüben (Krämpfe, Lähmungen, Veränderungen des Pulses und der Atmung u. s. w.). Manche bislang rätselhafte viscerale Störungen der Hysterie (zir-kulatorische, respiratorische, sekretorische), für welche eine psychische Entstehungsursache nicht nachzuweisen ist, sind solche Dauerwirkungen der Affekte. 
Sehr lebhaft tritt bei den hysterischen Gefühlsschwankungen das KontrastgefiiM zutage, besonders aber trifft man oft heitere, traurige, reizbare Stimmung, Mischgefühle, Spannungs- (Furcht) und Lösungsgefühle.

Es treten nun die merkwürdigsten und scheinbar widerspruchvollsten Beziehungen zwischen Gefühlserregungen und Organempfindungen auf, die unter dem Gesetze der Irradiation uud Reflexion, der Gefühlstöne verständlich werden.

Ausserordentlich wichtig und interessant ist das 2. Kapitel (Seite 138), in welchem der Verfasser die Empfindungsstörungen der Haut abhandelt. Er teilt dieselben ein in Hyp-, An- und Hyperästhesien und weist denselben mit folgenden Worten eine hohe Bedeutung zu: Die Störungen der Empfindungen und Gefühlsreaktionen gehören zu den wichtigsten Krankheitserscheinungen der Hysterie, und ihre genaue Peststellung ist für die Sicherung der Diagnose unerlässiich.

Er bespricht die Methodik der Sensibilitätsprüfung und verweist auf den Nutzen der Prüfung bilateraler, symmetrischer Hautstellen hin.

Betreffs der klinischen Bedeutung der Hautanästhesien, die in geradezu mustergültiger Weise geschildert werden, .nimmt der Autor einen Standpunkt ein, der mit dem des Referenten sich völlig deckt. Wie die statistischen Zusammenstellungen ergeben, gehören die Hautanästhesien sicherlich zu den Jiänfigsten Krankheitserscheinungen der Hysterie im interparoxystischen Stadium, sie-können also bei den Kranken die Feststellung der Diagnose zu Zeiten sichern, in welchen markante paroxystische Krankheitsäusserungen fehlen und der Bewusstseinszustand der Patienten zuverlässige Prüfungen der Empfindungen zulässt." Ausdrücklich hält Binswanger diesen Standpunkt trotz der neuerlichen Behauptungen Böttigers im ärztlichen Verein zu Hamburg in vollem Umfange aufrecht. Böttiger, dem im Verein Dr. Nonne sowohl wie Referent entgegengetreten waren, glaubt, dass sowohl die Ilcmianästhesic als auch die konzentrische Gesichtsfeldeinengung Kunstprodukte seien, die entweder auf Autosuggestion seitens der Kranken oder auf Fremdsuggestionen durch den Untersucher zurückzuführen seien. Nach Binswangers Ansicht hat Böttiger augenscheinlich diese Überzeugung durch die Untersuchung eines höchst einseitigen Materials gewonnen, vornehmlich an inveterierten Hystericis, die schon häufig Insassen von Nervenabteilungen gewesen waren.

Aus praktischen Gründen empfiehlt Binswanger die Untersuchung der elektrokutanen Empfindlichkeit, da es Fälle von isolierter Elektroästhesie gibt (Pitres, von Raben au).

Nachdem die Hyperästhesien und die Empfindungsstörungen der Schleimhäute geschildert sind, geht B. ausführlich auf die sensoriellen Empfindungsstörungen ein besonders auf die optischen. Die hysterische Amaurose ist eingehend dargestellt und besprochen.

Bezüglich der konzentrischen Gesichtsfeldeinschränkung bei der Hysterie möchte Referent aus seinen vielfältigen Erfahrungen und seiner jahrelangen Beschäftigung in der Wilbrandschen Augenpoliklinik mehr, als der Autor es tut, die pathognomonische Wichtigkeit dieses Symptoms hervorheben. Die Versuche von Schmidt-Rimpler, Vogel und Salomonsohn sind angestellt worden zu einer Zeit, in welcher die Frage der traumatischen Neurosen mehr oder weniger heftig J£örtert wurde, so dass oft mit einer gewissen Voreingenommenheit an die Untersuchung der Traumatiker herangegangen worden ist, wie dies besonders aus der vom Autor herangezogenen Abhandlung von Salomonsohn hervorzugehen scheint. In sämtlichen erwähnten Arbeiten waren die veränderten Adaptationsvorgänge bei der Hysterie gar nicht oder nicht genfigend in Rechnung gezogen; dagegen wird dem so leicht kontrollierbaren Faktor der Aufmerksamkeit eine übermässige Bedeutung beigemessen. Jeder erfahrene Augenarzt hat seit Einführung des Perimeters gelernt, diesen Faktor auszuschalten, und es würde zu weit gehen, anzunehmen, dass Männer wie Landolt, Charcot, Parinaud, Fere sich einer derartigen Unterlassungssünde schuldig gemacht hätten. 
Hinsichtlich des Satzes 216: ,dagegen kann nicht zugegeben werden, dass derartige Befunde (Ermüdungseinschränkung, Verschiebungstypus) eine pathologisch erhöhte Ermüdbarkeit der peripheren Sinnesapparate beweisen,, sie können ebenso gut mit der Auffassung in Einklang gebracht werden, dass hier kortiko-sensorische Ermüdung vorliegt oder dass es sich um Störungen der Aufmerksamkeit handelt", ist einfach auf die fundamentale Tatsache der Verlang sam ung des Adaptationsvorganges (Erholung der Netzhaut) zurückzuführen, den Dr. Wilbrand in einer Schrift „Die Erholungsausdehnung des Gesichtsfeldes unter normalen und pathologischen Bedingungen", Wiesbaden, Bergmann 1896, aufs eingehendste geschildert hat, und deren Anführung wir leider vermissen.

Das Charakteristische und daher pathognomonisch Wichtige der kon zentrischen

Gesichtsfeldeinschränkung bei Hysterie besteht nach des Referenten Ansicht darin, dass die Hysterischen kein Bewusstsein von dem Bestehen einer Einschränkung des Gesichtsfeldes haben und $\mathrm{n}$ i e mals darüber Klage führen.

Sehr selten ist völlige hysterische Taubheit, von der Binswanger ein charakteristisches Beispiel mitteilt.

Im 2, Kapitel behandelt Binswanger den hysterischen Schwindel, die S törung en der tieferen Sensibilität, die visceralen Empfin-dungsstörungen, die kutanen Analgesien und die hysterischen Hyperal-gesien. Es würde nun zu weit führen, auf die einzelnen Kapitel in der Weise einzugehen, wie Referent dies bisher getan hat; es sollen nur noch hervorgehoben werden die sehr lesenswerten Auseinandersetzungen B.s über die Headschen Zonen, die Akinesia algera und die Druckschmerzpunkte. Binswanger wendet sich gegen die Auffassung der französischen Schule von der Bedeutung der hysterogenen Zonen. Einmal gäbe es eine grosse Zahl ausgeprägter Hysterien, bei welchen die paroxystischen Erscheinungen an Häufigkeit und Bedeutung gegenüber den interparoxystischen weit zurück treten oder sogar völlig fehlen und bei denen trotzdem die charakteristischen Schmerzdruckpunkte nachzuweisen sind. Sodann gibt es Fälle mit voll entwickelten hysterischen Paroxysmen, bei denen jeder krampferzeugendo oder krampfhemmende Einfluss, falls alle Suggestion vermieden wird, durch Reizung der Druckschmerzpunktc resp. Zonen ausgeschlossen ^yerden kann.

Binswanger macht darauf aufmerksam, dass bei der Hysterie die Tendenz zu einer nemilateralen Anordnung der Druckpunkte besteht. Praktisch sehr bedeutsame Winke gibt der Autor betreffs der Erkenntnis des hysterischen Charakters mancher Fälle von Ischias, Migräne, von Kopfschmerzen, Arthralgie, Angina pectoris, von Enteralgien und Schmerzanfällen im Gebiete der Genital Organe.

Die Halluzinationen, welche sich bei den Hysterischen vornehmlich in hypnoiden Visionen kundgeben, werden durch eine charakteristische Krankengeschichte illustriert. Recht häufig werden bei Hysterischen auch illusionäre Umdeutungen wirklicher Empfindungen beobachtet. Sehr lesenswert ist der Abschnitt über die Störungen der Ideenassoziation der Hysterischen. In erster Linie ist die Konzentration der Aufmerksamkeit gestört; ferner ist von Bedeutung die Ers ch wer ung der Reproduktion der Erinnerungsbilder, welche mit den Gedächtnisstörungen eng zusammenhängen. Es ist fast immer die intellektuelle Erschöpfung das sinnfälligste Merkmal.

Mit der Angabe Binswangers, jene Personen als hysterisch anzusehen, bei welchen die Reproduktion einzelner, vor allem optischer Erinnerungsbilder erschwert sei, kann Referent jedoch nicht übereinstimmen, da er Patienten mit reiner Neurasthenie kennt, die nicht imstande waren, sich die Gesichter der nächsten Angehörigen vorzustellen.

Sehr instruktiv sind die Beispiele, die B. für das sogen. Wach-•txAtionen anführt, als deren Ursache er emotionellen Shock auf ein neuro-pathisch prädisponiertes Individuum (Alkoholismns, Gewerbekrankheiten, konstitutionelle Anämie) ansieht. 
Wichtig ist der von Binswanger aufgestellte Satz, dass dem hysterischen Symptomenkomplex auch Bewusstseinslücken, welche von denjenigen der epileptischen Erkrankung nicht zu unterscheiden sind, eigentümlich seien.

Die hysterische Launenhaftigkeit, die man jedoch nicht als patho-gnomonische Erscheinung der Hysterie bezeichnen soll, die hysterischen btimmungsanomalien, die zwangsweise Reproduktion von Erinnerungsbildern, die pathologische Lügenhaftigkeit, die phantastische Ausschmückung und Übertreibung der krankhaften Beschwerden werden von Binswanger mit einer Meisterschaft geschildert, wie wir sie bisher in einem deutschen Werke nicht gefunden haben. Die die jeweiligen Krankheitsbilder illustrierenden Krankengeschichten zeichnen sich durch prägnante, plastische Abfassung aus.

B. hebt aber hervor, und zwar, wie auch Referent meint, mit vollem Recht, dass es einfache, nicht degenerative Hysterien gibt, welchen die obengenannten Charakteranomalien völlig abgehen.

Zum Schluss des Kapitels bespricht B. den Selbstmord bei Hysterie und warnt davor, der unter Ärzten so sehr verbreiteten Ansicht zu sehr.zu vertrauen, dass Selbstmordversuche der Hysterischen nicht ernst genommen zu werden brauchen, denn nicht selten haben Hysterische in schweren Angstaffekten oder aus Wut, Trotz und Stolz das Suicidium, mit dem sie oft monatelang kokettiert haben, ausgeführt.

Den II. grossen Abschnitt bilden die somatischen Krankheitserscheinungen: 1. die Störungen der Motilität. Dieselben zerfallen in Lähmungen und Krämpfe.

Die auch von Binswanger anerkannte, von Charco t früher bestrittene hysterische Facialislähmung wird ausführlich geschildert, ebenso die Ptosis spastica und paralytica. B. schliesst sich der vom Referenten und Wi 1 b r $n$ nd gegebenen Erklärung an.

Binswanger führt die Literatur der hysterischen Lähmungen einzelner Augenmuskeln ziemlich vollständig an. Er selbst hat nie eine isolierte hysterische Augenmuskellähmung gesehen.

Die sonstigen hysterischen Lähmungen, die Kombinationen von Paresen und Spasmen werden in eingehender Weise besprochen; die pseudospastische Parese mit Tremor, die Fürstner 1896 zuerst beschrieben hat, rechnet Binswanger zu den neuropathisch-hypochondrischen Fällen; die Nonneschen Fälle dieser Art, die Ref. gesehen nat, sind wohl zur Hysterie zu rechnen.

Aus der Zusammenstellung der zahlreichen Fälle von Abasie und Astasie geht hervor, dass dieses Krankheitsbild nicht ausschliesslich durch den Ausfall bestimmter Bewegungskombinationen erklärt werden kann, vielmehr seien Tremor, ataktische Störungen oft von wesentlicher Bedeutung für das Zustandekommen der Abasie und Astasie.

Die hysterische Aphonie und der hysterische Mutismus werden in vorzüglichen Krankengeschichten geschildert. Als auslösende Ursachen sind in erster Linie gemütliche Erregungen zu nennen.

Die von Pitres zuerst geschilderte Pseudotabes hysterica, bei der die Patellarreflexe immer erhalten sind, ist eine sehr seltene Krankheitserscheinung. Häufiger wird das hysterische Stottern beobachtet.

Der hysterischen Kontraktur ist ein langer Abschnitt gewidmet, in welchem die traumatische und posttraumatische Kontraktur, sowie die Scoliosis hysterica eingehend geschildert werden.

Bei den intermittierenden Muskelkrämpfen geht B. auch auf die Streitfrage betr. der Myoklonie ein. Binswanger schliesst sich Un-verricht an, dass es eine kleine Gruppe von Krankheitsfällen gäbe, welche hinsichtlich ihrer Entwicklung, ihres Verlaufs und ihres Ausganges eine eigenartige Stellung einnehmen. Die Mehrzahl der als Myoklonie beschriebenen Fälle gehört sicherlich der Hysterie an. Ganz ähnlich liegen die Beziehungen zwischen der Maladie des tics convulsifs und der Hysterie.

Die Lach- und Weinkrämpfe, der Tremor finden eingehende 
Buchanzeigen. 
Berücksichtigung. .Von letzterem sind zahlreiche Kurven und Schriftproben zur Illustration des Gesagten beigegeben.

Im 2., 3 und 4. Kapitel werden die Spasmen des Respirationsapparates, des Intestinaltraktus und des CJrogenitalapparates erschöpfend dargestellt. Keferent kann aus eigener Erfahrung die Angaben B.s bestätigen, dass das hysterische Erbrechen eine lebensbedrohende Krankheitserscheinung werden kann. Er sah in zwei einschlägigen Fällen den Exitus eintreten. Sehr aktuelles Interesse hat die auf Seite 570 gegebene Krankengeschichte eines Falles von Pseudoappendicitis.

Die angio- und trophoneurotischen Störungen werden eingehend abgehandelt. Als bemerkenswert möchten wir hervorheben, dass B. keine reinen Fälle von Hautblutungen bei Hysterie gesehen hat und dass er der Stigmatisation sehr skeptisch gegenüber steht. Die Schleimhautblutungen der Hysteriker dagegen sieht B. als auf reellem Boden stehend an.

Ganz ausserordentlich lehrreich sind die mitgeteilten, von Stintzing beobachteten Fülle von sogen, hysterischer Anurie oder vielmehr Psoudo-anurie, aus denen hervorgeht, wie leicht man von Hysterischen getäuscht wird. Das trifft auch beim hysterischen Fieber zu, weshalb Strümpell, S chultze und Jolly überhaupt das Vorkommen desselben bestreiten.- Auch B in s w a n g er war nie in der Lage, einen unzweideutigen Fall zu beobachten.

Wichtig sind die Aeusserungen B.s betreffs der Reflexe. Er hebt hervor, dass die Bauch-, Plantarund Gaumenrefl exe bei vielen gesunden Individuen bald halb-, bald doppelseitig fehlen können. Daher legt B. auch kein allzugrosses Gewicht auf das Verhalten des Gaumenreflexes.

Niemals hat Binswanger bei Hysterie das Babinskische Phänomen beobachtet, auch wenn dieselbe mit ausgeprägten spastischen Erscheinungen der Beinmuskulatur verbunden war.

Auf den Verlust des Schlund-, Würg- und Lidreflexes legt B. nicht solchen Wert, wie die Franzosen es tun. Er hält auch den Lidschlussreflex für ein wenig verlässliches Mittel zur Feststellung der Diagnose Hysterie.

Die Sehnenphänomenc sind bei der Hysterie sehr häufig gesteigert. B. hält trotz der Angaben in der Literatur (Nonne, Marie, Souza-Seite, Dejerine u. A.) daran fest, dass ein Verlust der Sehnenphänomene bei der hysterischen Lähmung nicht vorkomme. Referent schliesst sich auf Grund seiner Erfahrung B. durchaus an. Er hat noch niemals ein Fehlen der Sehnenreflexe bei Hysterie beobachtet. Ebenso stimmt Referent durchaus B. in dem bei, was er in seinem sehr lesenswerten Kapitel über die hysterischen Pupillenstörungen sagt, dass die reflektorische Pupillenstarre kein Symptom der Hysterie sei, sondern in die Domäne der organischen Gehirn-und Rückenmarksleiden gehöre. Sehr interessante und lehrreiche kasuistische Beiträge erhärten diese Ansicht.

Referent möchte hinzufügen, dass man erst von einer Pupillenstarre sprechen kann, wenn man auch mittels Westienscher Lupenbetrachtung keine Bewegung der Iris mehr nachweisen kann.

Die hysterischen Paroxysmen werden mit Beibringung vieler eigenbeobachteter Fälle ausführlich beschrieben. Der ausgebildete hysterische Anfall lässt 3 scharf getrennte Stadien nach B. unterscheiden:

1. das prodromale,

2. das konvulsivische,

3. das Nachstadium.

Im 2. Kapitel dieses Abschnittes werden die Aequivalente der hyste $\wedge$ rischen Paroxysmen besprochen.

1. Der synkopale Anfall, welcher meist 5 bis 10 Minuten dauert (Abarten sind der hysterische Schwindel und die sogen. Lethargie),

2. die hysterischen Schlaf anfälle,

3. die hystero-kataleptischen Anfälle,

4. die hystero-somnambulen Anfälle (hysterischer Dämmerzustand).

Bemerkenswert ist, dass die neueren Forschungen dargetan haben,

dass eine totale Amnesie nicht einmal in der Mehrzahl der Fälle

hysterischem Dämmerzustand nachgewiesen werden kann Forner komm etzterer auch unabhängig von anderweitigen paroxysmalen Krankheits- 
Äusserungen als akut einsetzender Krankheitszustand vor. Bei dem hysterischen Dämmerzustande handelt es sich nach Binswanger um eine traumhafte ßewusstseinsverminderung durch tiefgreifende Störung der Ideenassoziation. Das interessante Faktum des Wandertriebes (Poriomanie) auf hysterischer Grundlage wird eingehend behandelt. Durch zahlreiche eigene kasuistische Beiträge werden die hysterischen Dämmerzustände illustriert.

Was die namentlich von französischen Autoren aufgestellte Lehre von den Variationen der Persönlichkeit betrifft, so hält B. weder das wissenschaftlich gesicherte Beobachtungsmaterial, noch die daran sich anschliessenden psychologischen Deduktionen für ausreichend begründet, um aus derartigen Bewusstseinsveränderungen den kühnen Schluss auf Verdoppelungen der Persönlichkeit ziehen zu können.

In Bezug auf das Gansersche Symptom des Vorbeiredens bestätigt B. die Ansicht A. Westphals, dass dieses Symptom hei verschiedenartigen psychischen Störungen vorkommt.

Was die hysterische halluzinatorische Verwirrtheit betrifft, so ist es, wie B. hervorhebt, schwierig, dieselbe strikte von den somnambulen, resp. Dämmerzuständen zu scheiden; als treffendes Beispiel wird die Krankengeschichte No. 100 angeführt.

Den Schluss dieses interessanten mit packender Lebendigkeit geschilderten Kapitels bilden die von P. Richer beschriebenen Anfälle von Kontraktur.

Das 3. Kapitel beschäftigt sich mit dem grossen Anfall der Char-cotschen Schule, welcher sich durch den protrahierten Verlauf und die Mannigfaltigkeit der psychischen Krankheitserscheinungen auszeichnet.

Eine höchst ausführliche eigene Krankengeschichte leitet die sich anschliessenden Bemerkungen und Wiedergabe der einzelnen einschlägigen Abschnitte des Werkes von P. Ilicher ein. Es muss aber hervorgehoben wei'den, dass B. den Verdiensten der Schule Charcots um die Erforschung und die klinische Würdigung der einzelnen Glieder des grossen hysterischen Anfalls zwar sehr hohe Anerkennung zollt, dass er aber den überall betonten Grundgedanken für unrichtig hält, dass sich die zahllosen Varietäten der grossen und kleinen Anfälle in gesetzmässige Beziehungen zu dieser oder jener Periode des grossen Anfalls bringen lassen. Referent stimmt darin B. durchaus zu.

Anhangsweise werden die hysterischen Psychosen auf einigen Seiten besprochen. Man trifft hier Melancholie, maniakalische Exaltation, akute und chronische Paranoia, Dementia praecox.

Im Abschnitte IV wird die allgemeine Psychopathologie der Hysterie eingehend behandelt. Die theoretischen Anschauungen Janets, auf Grund seiner feinsinnigen Beobachtungen an den schweren degenerativen Hvsteriefällen der Pariser Krankenhäuser, hält der Autor nicht als massgebend für das gesamte weite Gebiet der hysterischen Veränderung. Letztere hat Binswanger schon im 1. Kapitel zu erklären versucht. Ganz besonders ausführlich beschäftigt sich der Autor mit den bekanntenBreuer-Freundschen Hysterieforschungen, speziell auch mit der Lehre der hysterischen Konversion, kommt aber zu dem auch vom Referenten geteilten Schluss, dass die Breuer-Freundsche Auffassung zu einseitig sei.

Der V. Abschnitt des Werkes enthält den Verlauf, die Prognose und Diagnose der Hysterie.

B. unterscheidet die le i cht er e (vulgäre) und die schwere (degenerative) Hysterie.

Der Verlauf der vulgären Hysterie wird im wesentlichen bestimmt

a) von der besonderen Art und dem Masse der affektiven Erregbarkeit und

b) von der Häufigkeit und Beschaffenheit der hysterischen Anfälle.

Auf der Grenze zwischen den mittelschweren und schwersten Formen der Hysterie stehen diejenigen Fälle, bei welchen sich auf Grund elementarer hysterischer Empfindungs- und Bewegungsstörungen sekundäre hypochondrische Krankheitszustände entwickelt haben, meist mit Zwangsvorstellungen verknüpft. 

Buchanzeigen. 
Die schwersten Fälle, die grosse Hysterie, sind durch die zusammengesetzten konvulsivischen und psychischen Anfälle ausgezeichnet.

Die sogen, monosymptomatische Form der Hysterie gehört zu den affektiven Hysterien mittleren Grades und kommt am meisten bei Kindern vor.

Was die prognostische Frage betrifft, so bejaht B. die Heilbarkeit im Hinblicke auf die kindliche und juvenile Hysterie. Bei den Erwachsenen handelt es sich mehr um die Beseitigung einzelner Zustandsbilder.

Die Prognose der Hysterie, quoad sanationem completam, ist in der Mehrzahl der Fälle eine ungünstige.

Ob die Hysterie als solche letal endigen kann, hält B. im allgemeinen für zweifelhaft, gibt dies aber zu für die Fälle von langdauernden Anorexien mit hysterischem Erbrechen. Ref. hat, wie gesagt, 2 solcher Fälle letal endigen sehen.

Zum Schlüsse dieses Kapitels bespricht Binswanger noch die Komplikationen der organischen Hirnerkrankungen mit Hysterie, das Verhalten der Pupillen im hysterischen Anfalle, die Mischformen der Hysterie und Epilepsie und die Frage des Zusammenhanges der Hysterie mit Genitalerkrankungen. Erfreulicherweise wendet sich der Autor gegen den bekannten Aufsatz von W.A.Freund und gegen einige Berliner Frauenärzte und bezieht sich auch auf die Gynäkologen Olsh a us en, Lomer und Krönig, die auf modern neurologischem Standpunkte stehen.

Betreffs der S i mulation der Hysterie spricht B. aus, dass seine eigenen Erfahrungen ihm die Ueberzeugung verschafft haben, dass die Simulation dos Gesamtleidens Hysterie kaum jemals versucht werde, geschweige denn gelungen sei.

Im Abschnitte VI legt B in s w a n g e r seine reichen Erfahrungen betreffs der Therapie der Hysterie nieder.

Die Psychotherapie, die diätetisch-physikalische, die arzneilichen und lokaltherapeutischen Behandlungmethoden werden in der ausführlichsten und instruktivsten Weise besprochen, so dass Referent wünschte, jeder Arzt würde sich mit diesem Kapitel vertraut machen. Sehr erfreulich war es dem Referenten, zu sehen, dass der Autor die Hypnotherapie nur als einen kleinen Abschnitt der Psychotherapie bezeichnet. Sie soll nur unter ganz bestimmten Voraussetzungen und bei besonders dazu geeigneten Fällen verwendet werden.

Durch ein alphabetisches Sachregister ist die praktische Brauchbarkeit dieses grossen Werkes über Hysterie ausserordentlich erhöht worden, mit dem Binswanger eine empfindliche Lücke in der deutschen medizinischen Literatur in meisterhafter Weise ausgefüllt hat.

Nagel, W., Handbuch der Physiologic des Menschen. Braunschweig. 1904 u. 1905. §c. Vieweg und Sohn. DasHerrma nn sehe Handbuch ist durch die physiologischen Forschungen der letzten Jahrzehnte auf fast allen Gebieten längst überholt worden. Es war deshalb schon immer wieder gefragt worden, oh trotz des fortgesetzten Zuströmens neuer Arbeiten nicht endlich die Zeit gekommen sei, abermals eine Gesamtdarstellung des Bestandes unseres physiologischen Wissens zu geben. Dieser Wunsch soll jetzt erfüllt werden. Die bis jetzt vorliegenden vier Teilbände versprechen allen billigen Anforderungen, welche man an ein solches Sammelwerk stellen kann, gerecht $\mathrm{zu}$ werden. Im ersten Teijband behandelt Boruttau „Die Atembewegungen und ihre Innervation", Bohr „Die Blutgase und den respiratorischen Gaswechsel", F. B. Hofmann „Die allgemeine Physiologie des Herzens" und „Die Innervation des Herzens und der Blutgefässe". Der zweite und dritte Teilband sind der Physiologie der Sinne gewidmet, die damit bereits abgeschlossen ist. Bei den Neuropatho-logen wird sich gerade dieser Teil sehr bald als ganz unentbehrlich erweisen. Speziell möchte ich auf die ausgezeichnete Darstellung der Lehre von den Gesichtsempfindungen durch .1. v. Kries und der Lehre von den Augenbewegungen und Gesichtswahrnehmungen durch 0. Zoth aufmerksam machen. Auch die Kapitel über den Gehörssinn (K. L. S c häf er), über den 
Geschmacks- und Geruchssinn (W. Nagel) und über die Unästhetischen Empfindungen (W. Nagel) sind ganz vortrefflich. Ueberall finden sich sorgfältige, wenn auch nicht vollständige Literaturangaben. Der vierte Teil band behandelt die erste Hälfte der Physiologie des Nerven- und Muskel systems und darf daher auf ganz spezielles Interesse bei den Neurologen rechnen. Bis jetzt liegt vor die Physiologie des Gehirns von Tschermak, die Physiologie des Kücken- und Kopfmarks von Langendorff und die Physiologie des sympathischen Nervensystems von P. Schultz. Auch diese Abschnitte sind mit ganz vereinzeltan Ausnahmen, zu denen ich beispiels weise die Darstellung der Aphasielehre rechne, klar und übersichtlich ge schrieben und geben eine sehr korrekte und fast vollständige Darstellung unserer jetzigen Kenntnisse auf diesem Gebiete. Die Ausstattung ist sehr gut, der Druck sehr übersichtlich. Die Abbildungen könnten etwas zahl reicher sein. Es ist zu hoffen, dass der Verleger die für den Neurologen speziell in Betracht kommenden Bände III und IT auch einzeln abgeben wird. Soviel lässt sich schon jetzt sagen: das neue Handbuch wird sich dem Herrmannschen ebenbürtig an die Seite stellen dürfen.

Berger, Hans, Privatdozent der Psychiatrie in Jena: Heber die körperlichen Aeusserungen psychischer Zustände, Jena 1904. Verlag von G. Fischer.

Nach einem geschichtlichen Ueberblick über die Untersuchungen der Zirkulationsverhältnisse im Schädel und deren Beeinflussung durch Gemütsbewegungen geht Verfasser zur Beschreibung der Untersuchungsmethoden über. Er bediente sich der Ausdrucksmethode mit der Anwendung äusserer Reize.

Verfasser wendet sich dann der Lehre von den Gefühlen zu, die er mit der Mehrzahl der von ihm angeführten Autoren als selbständige Be-wusstseinselemente auffasst und mit Wundt als die subjektiven Bewusstseins-inhalte definiert. Unter Affekten versteht er nur solche Bewusstseinsvorgänge, die Gefühle enthalten, sich jedoch durch ihre Intensität von den einfachen Gemütsbewegungen unterscheiden, während er die Stimmung als einen chronischen Gefühlszustand ansieht.

Für seine Versuche kommen hauptsächlich die zentralphysiologischen Theorien in Betracht. Auf die Arbeiten von Lehmann und wundt gestützt, hält Verfasser den Energieumsatz der einzelnen im Apperzeptionszentrum gelegenen Neurone für die materielle Grundlage der Gefühlsvorgänge.

Verfasser schreitet nunmehr zur Mitteilung seiner VersucEe. Als Versuchsperson diente ihm ein Arbeiter, bei dem 1897 eine Trepanationsöffnung am rechten Parietale zwecks Entfernung einer Kugel angelegt war.

Darauf folgt eine genaue Beschreibung der für die Versuche benutzten Instrumente, sowie eine Darstellung der Versüchsanordnung und der Bearbeitung des gewonnenen Materials. Die Kurven sind, so geschrieben, dass oben die Respirationskurve, in der Mitte die plethysmographische Kurve des Arms und unten das Gehirnvolumen verzeichnet ist.

Es wird nun der Einfluss der Aufmerksamkeit, die in willkürliche Aufmerksamkeit, Erschrecken und Spannung unterschieden wird, der Gefühle und des Schlafes auf jede der 3 Kurven beschrieben.

Als physiologische Ursache der Zu- und Abnahme der Volumkurve des Gehirns nimmt Verfasser bei Konzentration der Aufmerksamkeit und psychischer Arbeit eine aktive Erweiterung, beim Schreck eine aktive Kontraktion der Gefässe an.

Weiterhin führt er aus, dass unlustbetonte Empfindungen mit einer Kontraktion, lustbetonte mit einer Erweiterung speziell der Rindengefässe einhergehen, und fasst diese Veränderungen des Gefässtonus als Folgeerscheinungen der mit den Gefühlsvorgängen verbundenen kortikalen Prozesse auf.

Schliesslich gelangt er unter Zugrundelegung der Verwornschen Biogenhypothese $\mathrm{zu}$ der Auffassung, dass das VerWßnis der Assimilations--Prozesse zu den Dissimilationsprozessen in der Hirnrinde, der sog. Biotonus Sie Weite der dieselbe versorgenden Gefässe bestimmt.

B. Pfeifer-Halle a. S. 